\title{
Broken Epidural Catheter: An Anesthesiologist's Dilemma
}

\author{
Dinesh Kumar Sardana, Karan Panaych, Tanvir Samra \\ Department of Anesthesia \& Intensive care, Post Graduate Institute of Medical Education and Research, Chandigarh-160012, India.
}

\section{Corresponding Author:}

Dr. Dinesh Kumar Sardana

Email: dineshsardana24@yahoo.com

This is an Open Access article distributed under the terms of the Creative Commons Attribution License (creativecommons.org/ licenses/by/3.0).

Received : October 12, 2016

Accepted : January 23, 2017

Published : March 25, 2017

\begin{abstract}
Epidural anesthesia is a safe procedure and is routinely performed by the anesthesiologists. Breakage of an epidural catheter is a rare, but a worrisome complication. However, if this happens, the presence of retained epidural catheter fragment should be properly documented and should also be informed to the surgical team and the patient. Here, we present two cases of such an event and also highlighting the common reasons that could have precipitated that event.
\end{abstract}

Keywords: Analgesia, Anesthesia, Bupivacaine, Catheterization, Choledochal Cyst, Epidural Space.

\section{Introduction}

Epidural catheterization is a commonly performed procedure in anesthesia. The insertion of an epidural catheter, although safe, has been known to break during removal, leaving a segment lodged in patient's back [1]. Sequestered catheter fragment is generally considered to be inert and should not produce a foreign body reaction. Therefore, in most of the cases, it is recommended to leave the catheter fragment alone, unless symptomatic, as the surgical intervention would do more harm than good [2].

\section{Case Report}

\section{Case 1}

A 27 year-old-male was admitted with a diagnosis of bilateral upper ureteric stone and was planned for bilateral ureteroscopic stenting plus bilateral retrograde intra-renal scanning (B/L URS plus $B / L$ RIRS) under combined spinal - epidural anesthesia (anticipating prolonged procedure). An 18 gauze, $80 \mathrm{~mm}$ Touchy needle, (Portex EPI - KIT) was inserted in the L3-L4 intervertebral space with loss of resistance technique in the sitting position.
The epidural space was encountered at $5 \mathrm{~cm}$ from the skin and a 19 gauze radio-opaque multihole epidural catheter was advanced up to $9 \mathrm{~cm}$ after which resistance was felt and the catheter could not be threaded into the epidural space. Therefore, it was decided to relocate the epidural space. While the catheter was being removed with gentle traction along with the epidural needle, the blue tip got sheared off [Fig.1].

A new epidural catheter was inserted at L2L3 intervertebral space. The epidural catheter was fixed at $11 \mathrm{~cm}$. The sub-arachnoid block was given in the same space with $3 \mathrm{ml}$ of $0.5 \%$ bupivacaine (heavy) and $25 \mu \mathrm{g}$ fentanyl. The patient was

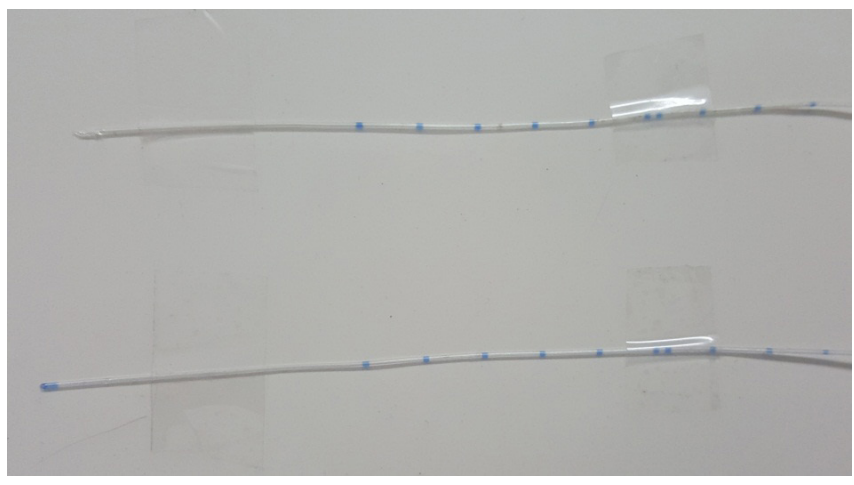

Fig.1: Broken epidural catheter (blue tip missing). 
placed in Trendelenburg position. The surgery was started after checking the sensory level (T6). The total duration of the surgery was two and a half hours. Intra-operatively, a single top up dose of $0.5 \%$ bupivacaine $(6 \mathrm{ml})$ was given. T6 level was maintained throughout the procedure. Postoperatively, the analgesia was maintained with top up boluses of $10 \mathrm{ml} 0.125 \%$ bupivacaine given at 2 hourly intervals. The epidural catheter was removed 6 hours after the procedure. No sensory or motor deficit was noted four hours after its removal.

\section{Case 2}

A 10 year-old-female was admitted with a diagnosis of choledochal cyst and was planned for choledochal cyst excision and hepaticojejunostomy under general anesthesia plus epidural catheter. The patient was given general anaesthesia and placed in left lateral position for epidural catheterization. A 19 G Touhy needle was inserted in the L1-L2 space and after finding the epidural space using loss of resistance to saline technique, catheter insertion was attempted. However the catheter could not be threaded adequately (resistance was felt) and an attempt for removal of the same was made with minimal force. However on removal of the catheter the blue tip was not seen and the catheter was found to be broken at the $5 \mathrm{~cm}$ mark [Fig.2]. No further attempts were made for epidural catheter insertion and the surgery was started. The duration of the surgery was 3 hours. At the end of the surgery, patient was extubated uneventfully and shifted to the recovery room.

Both the patients did not have any feature of local infection, sepsis or any other neurological deficit. They were followed up till the day of discharge. X-ray lumbar spine (both antero-posterior and lateral) were done. No abnormality was detected. Since they did not have any neurological signs and symptoms, no further imaging was done. The patient and the patient's relatives were informed and counseled regarding

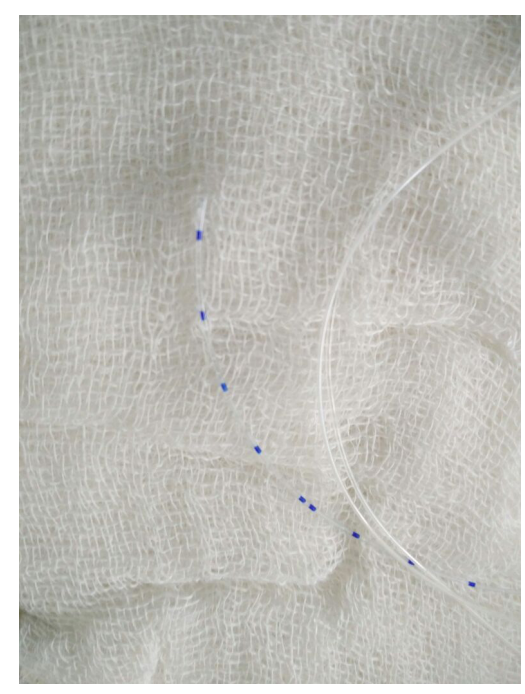

Fig.2: $5 \mathrm{~cm}$ broken epidural catheter.

the possible complications related to the retained epidural catheter fragment. They were kept on a regular follow up with an advice to report in case of any abnormal sign and symptoms. Both were found to be well after a follow up of 15 months.

\section{Discussion}

A broken spinal or epidural catheter, although an uncommon occurrence, remains an area of utmost dilemma to the practicing anesthesiologist. The possible causes of the broken epidural catheter could be: (i) catheter sliced into two pieces while withdrawing, (ii) application of undue force resulting in breakage, stretching or tearing of catheter, (iii) manufacturing defect of catheter, (iv) heavy contact between the tip of an epidural needle and the bony surface resulting in catheter breakage, (v) catheter damage due to excessive insertion [3-5].

Radiography tests are not very useful in diagnosing a sheared epidural catheter fragment. It is impossible to locate a radio-opaque fragment radiologically, because the surrounding structures are radio-dense. Paradoxically, the radio-opaque catheters have a lower tensile strength than the clear ones. In a study, a broken catheter becomes walled off by fibrous tissue after remaining in the 
epidural space for about 3 weeks [6]. Foreign body in epidural space is not likely to migrate (although this is not impossible). However, Stats et al. reported the formation of a reactive epidural mass $(1.5 \mathrm{~cm})$ around the catheter fragment resulting in lumbar spinal stenosis, patient being asymptomatic until 18 months of the incident. It got relieved with removal of catheter and reactive scar tissue [7]. Rare case of low back ache due to foramina stenosis has been reported [8]. In such cases, surgical intervention must be done for the removal of the catheter. Recently, Ishikawa et al. reported a case of delayed onset of subdural hematoma (18 years) following epidural catheter breakage, exploring the possibility of a relationship between catheter fragment and subdural hematoma. Decompression surgery was done to relieve the patient of symptoms [9]. If the proximal end of the segment is located at or just beneath the skin such that it can be retrieved through a skin incision by gentle traction [10]. Surgical removal is mandatory in such a situation as bacteria can readily track along the catheter remnant.

The patient was informed and counseled about the possibility of any complication relating to the retained epidural catheter fragment tip. He was asked to report in case of any adverse symptoms. Furthermore, the surgeon was also informed. The patient was followed for 15 months during which he remained symptom free.

\section{Conclusion}

The presence of a retained epidural catheter fragment tip must be addressed and communicated both to the surgeon and the patient. The patient must be counseled and given reassurance. Radiological imaging should be done at the earliest, in case the patient reports any neurological symptoms. Early surgical intervention to retrieve the retained catheter fragment is advocated (in case of neurological symptoms). Patients should be followed routinely as symptoms can occur even months or years later.

Contributors: DKS: Manuscript writing and literature review; KP: Manuscript revision; TS : Literature review. All authors were involved in case management. DKS will act as guarantor. All authors approved the final version of the manuscript.

Funding: None; Competing interests: None stated.

\section{References}

1. Tao T, Macmurdo S, McKenzie R. Mishap with an epidural catheter. Anesthesiology. 1979;50:260-262.

2. Metra R, Fleischmann K. Management of the sheared epidural catheter: is surgical extraction really necessary? Journal of Clinical Anesthesia. 2007;19:310-314.

3. Gough JD, Johnston KR, Harmer M. Kinking of epidural catheters. Anesthesia. 1989;40:1060.

4. Collier C. Epidural catheter breakage: a possible mechanism. Into J Obstetric Amnesty. 2000;9:87-93.

5. Dawkins M. An analysis of the complications of extradural and caudal block. Anesthesia. 1969;24:554563.

6. Bromate PR. In: Epidural Analgesia. Philadelphia, WB Saunders. 1978 pp. 664-666.

7. Stats PS, Stinson MS, Lee R. Lumbar stenosis complicating retained epidural catheter tip. Anesthesiology. 1995;83:1115-1118.

8. You JW, Cho YH. Foramina stenosis complicating retained broken epidural needle tip - A case report. Korean J Anesthesiology. 2010;59:69-72.

9. Ishikawa $\mathrm{Y}$, Magma $\mathrm{S}$, Ito Z, et al. Delayed onset of subdural hematoma following epidural catheter breakage. Global spine J. 2016;6(1):e1-6.

10. De Armendi AJ, Ryan JF, Chang HM, Liu LMP, Jaramillo D. Retained caudal catheter in a pediatric patient. Paid Anes. 1992;2:325-327. 